\title{
RTE and CTE mRNA export elements synergistically increase expression of unstable, Rev-dependent HIV and SIV mRNAs Sergey Smulevitch ${ }^{1}$, Jenifer Bear ${ }^{1}$, Candido Alicea ${ }^{1}$, Margherita Rosati ${ }^{2}$, Rashmi Jalah ${ }^{1}$, Andrei S Zolotukhin ${ }^{1}$, Agneta von Gegerfelt ${ }^{2}$, Daniel Michalowski ${ }^{1}$, Christoph Moroni ${ }^{3}$, George N Pavlakis ${ }^{2}$ and Barbara K Felber*1
}

Address: ${ }^{1}$ Human Retrovirus Pathogenesis Section, National Cancer Institute-Frederick, Frederick, MD 21702-1201, USA, ${ }^{2}$ Human Retrovirus Section, National Cancer Institute-Frederick, Frederick, MD 21702-1201, USA and ${ }^{3}$ Institut für Medizinische Mikrobiologie Universitaet Basel, Basel, Switzerland

Email: Sergey Smulevitch - smulevit@hotmail.com; Jenifer Bear - bear@ncifcrf.gov; Candido Alicea - calicea@ncifcrf.gov; Margherita Rosati - rosati@ncifcrf.gov; Rashmi Jalah - rjalah@ncifcrf.gov; Andrei S Zolotukhin - zolotukh@ncifcrf.gov; Agneta von Gegerfelt - vongeger@ncifcrf.gov; Daniel Michalowski - michalowskid@missouri.edu; Christoph Moroni -Christoph.Moroni@unibas.ch; George N Pavlakis - pavlakis@ncifcrf.gov; Barbara K Felber* - felber@ncifcrf.gov

* Corresponding author

Published: 13 January 2006

Retrovirology 2006, 3:6 doi:10.1186/1742-4690-3-6

This article is available from: http://www.retrovirology.com/content/3/l/6

(C) 2006 Smulevitch et al; licensee BioMed Central Ltd.

This is an Open Access article distributed under the terms of the Creative Commons Attribution License (http://creativecommons.org/licenses/by/2.0), which permits unrestricted use, distribution, and reproduction in any medium, provided the original work is properly cited.
Received: 07 November 2005

Accepted: 13 January 2006

\begin{abstract}
Studies of retroviral mRNA export identified two distinct RNA export elements utilizing conserved eukaryotic mRNA export mechanism(s), namely the Constitutive Transport Element (CTE) and the RNA Transport Element (RTE). Although RTE and CTE are potent in nucleocytoplasmic mRNA transport and expression, neither element is as powerful as the Rev-RRE posttranscriptional control. Here, we found that whereas CTE and the up-regulatory mutant RTEm 26 alone increase expression from a subgenomic gag and env clones, the combination of these elements led to a several hundred-fold, synergistic increase. The use of the RTEm26-CTE combination is a simple way to increase expression of poorly expressed retroviral genes to levels otherwise only achieved via more cumbersome RNA optimization. The potent RTEm26-CTE element could be useful in lentiviral gene therapy vectors, DNA-based vaccine vectors, and gene transfer studies of other poorly expressed genes.
\end{abstract}

\section{Background}

Posttranscriptional events determine the fate of cellular and viral mRNAs through concerted actions promoting nuclear trafficking and cytoplasmic transport, stabilization and translation. Simian type D (SRV/D) retroviruses and intracisternal A-particle retroelements (IAP) have provided us with unique mRNA transport elements, which utilize conserved cellular export machinery [1-13]. The export of the SRV/D unspliced mRNA is mediated by the cis-acting constitutive transport element CTE $[8,10-13]$ through interaction with the cellular NXF1 protein [1], which is also the key factor mediating general mRNA export [1-5], a property which is conserved among eukaryotes (reviewed in [14-16]). We previously identified another functionally similar but structurally unrelated posttranscriptional RNA Transport Element RTE [6,7], which is present in a subgroup of murine IAP. Both CTE and RTE utilize the conserved eukaryotic mRNA transport 


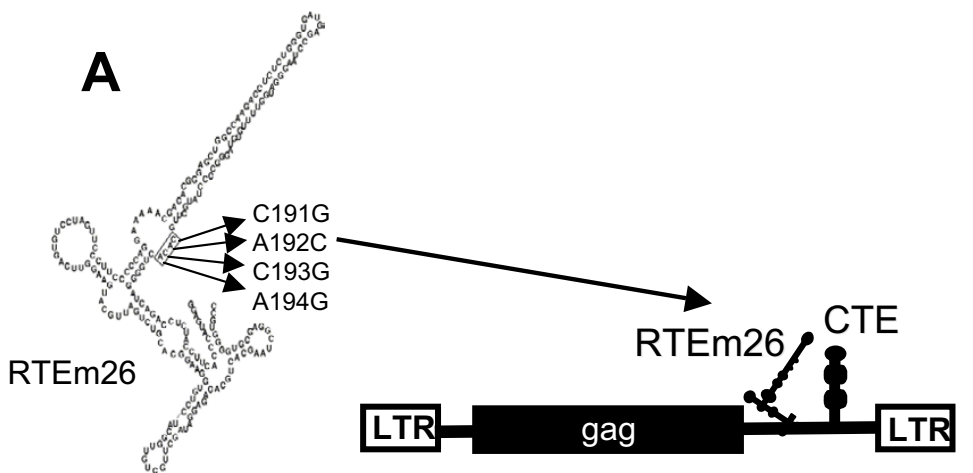

B

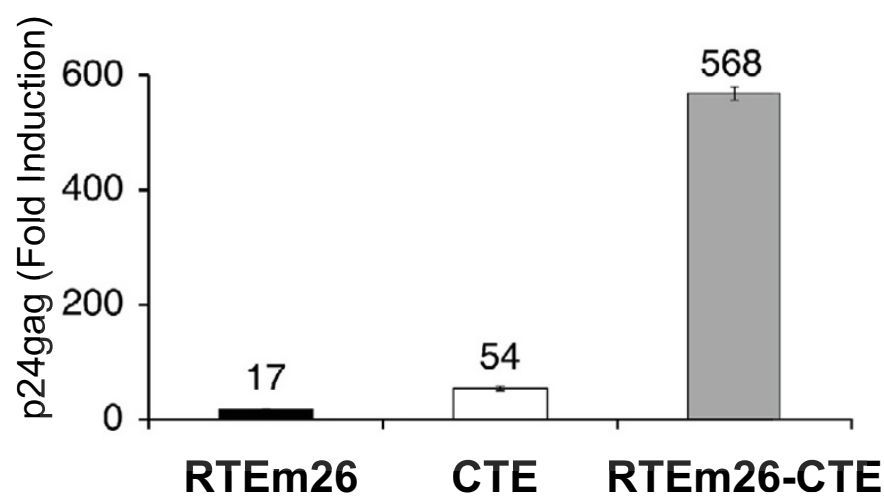

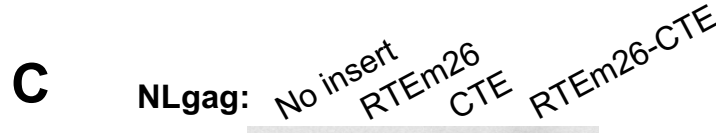

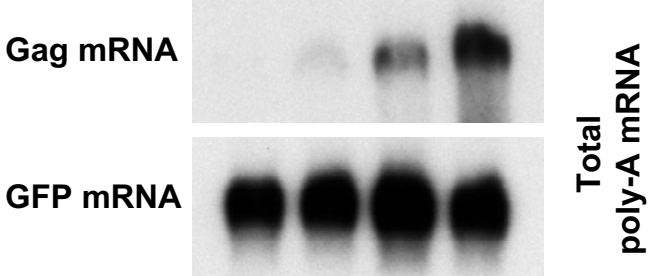

Fold increase in: $\begin{array}{lrrrr}\text { gag mRNA } & 1 & 4 & 12 & 29 \\ \text { Gag protein } & 1 & 13 & 78 & 557\end{array}$

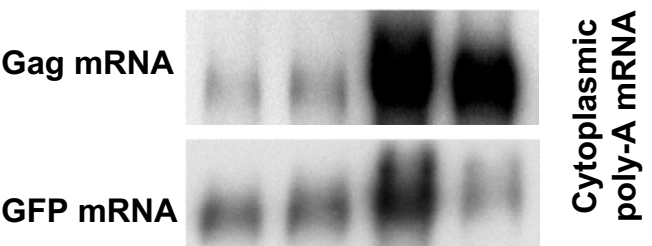

Figure I

RTEm 26-CTE is a potent combination of RNA transport elements. A) Structure of the gag reporter plasmid. The HIV-I gag gene is flanked by the $5^{\prime}$ and 3 'LTRs providing promoter and polyadenylation signals, respectively. NLgag contains the major splice donor of HIV-I located 5' to gag and a cryptic splice acceptor between RNA export elements and the 3'LTR and expresses HIV-I gag [23, 24, 39]. The RTE structure [7] shows the nucleotide changes in mutant RTEm26 (nt I90-I93 CACA changed to GCGG). The 226-nt RTE and the I73-nt CTE were inserted between the gag gene and the 3'LTR, generating the NLgagRTEm26-CTE. B) Expression of the gag reporter pNLgag plasmids, containing either no insert, RTEm26 or CTE alone, or the RTEm26-CTE combination. Cell extracts from transfected HeLa cells were analyzed for Gag production using an HIV-I gag antigen capture assay. Gag expression is presented as fold induction as compared to the gag levels produced by $\mathrm{pNLgag}$. Standard deviations are shown. C) Northern blots of total polyA-containing (top panel) and cytoplasmic (bottom panel) mRNAs from cells transfected with pNLgag or pNLgag containing RTEm26, CTE, or RTEm26-CTE were hybridized with a probe spanning the 3'end of the gag mRNAs [12]. Hybridization of the blot with a GFP probe serves as internal control of transfection efficiency and RNA preparation. The blots shown in the top and bottom panels are from two independent experiments. Note that the cytoplasmic poly-A mRNA samples are unequally loaded, and the CTE lane has 2.5-fold more GFP mRNA while the RTEm26-CTE lane has $60 \%$ of the GFP mRNA compared to the other lanes (no insert, RTEm26). The blots were quantitated using the STORM860 phosphoimager.

machinery. Here, we demonstrate that the combination of RTE and CTE in cis leads to synergistic increase in lentiviral gene expression.

\section{Results}

Synergistic activation of gene expression in the presence of a combination of RTE-CTE

Since the presence of RTE or CTE positively affects production of poorly expressed retroviral genes, we asked whether the RTE-CTE combination in cis has an additive or synergistic effect on gene expression. For this, we used the up-regulatory mutant RTE (RTEm26) (Figure 1A), known to increase RTE function by 2-fold [7], in combination with the SRV-1 CTE. The reporter plasmids used for these studies encode HIV-1 gag or env genes (Figures 1 and 2 ), which are known to be poorly expressed in the absence of a positive-acting posttranscriptional regulatory system [17-29]. In pNLgagRTEm26-CTE, the RTEm26 was inserted 5 ' to the CTE into reporter pNLgagCTE (Figure 1A). Upon transfection into human HeLa cells, we found that whereas RTEm 26 or CTE alone activated Gag production by $\sim 20$-fold and $\sim 50$-fold, respectively (Figure $1 \mathrm{~B}$ ) as 


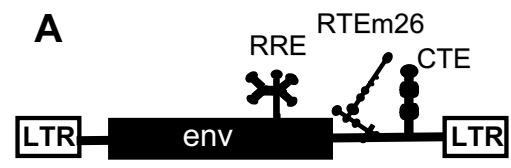

B

HIV-1 env cDNA clone pNL1.5E containing RNA export elements:

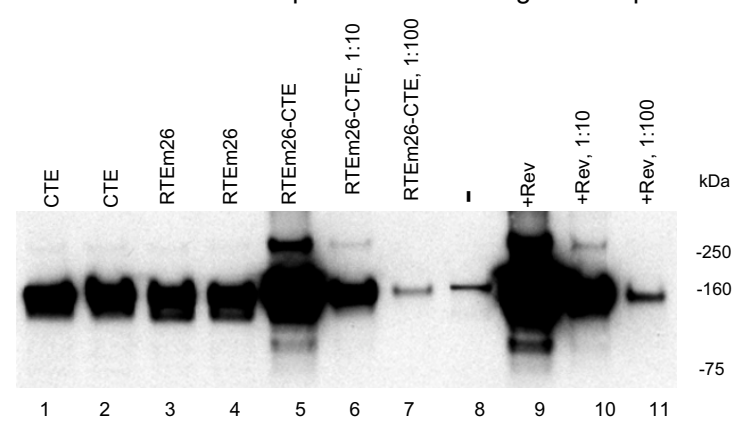

Figure 2

RTEm26-CTE synergistically increase HIV-I env production. A) The structure of the env cDNA plasmid PNLI.5E containing the RTEm26-CTE. The env gene contains the Rev-responsive element RRE within env and is expressed from the HIV-I LTR promoter. RTE, CTE and RTEm26-CTE were inserted between the env gene and the 3' LTR. B) HLtat cells were transfected with the indicated plasmids and analyzed for Env production by Western blot analysis using a rabbit anti-HIV-I env serum.

expected, the combination of these elements had a synergistic effect, leading to a dramatic 570-fold activation (Figure 1B). Synergy was only observed when the elements were present in cis, but not upon co-transfection of the RTE- and CTE-containing reporters within the same cells (data not shown). Similar data were obtained by using a splice donor-deleted gag reporter, pNLcgag [24], which only produces an unspliced gag mRNA [24]. This experiment suggests that the synergistic effect of RTEm26CTE is independent of splicing (data not shown). Analysis of total poly-A containing mRNAs from the transfected HeLa cells (Figure 1C) showed that the presence of either element alone elevated gag mRNA levels (4- and 12-fold, respectively) and the RTEm26-CTE combination resulted in a further increase (29-fold). Analysis of cytoplasmic mRNA (Figure 1C, bottom panel) confirmed that RTEm26-CTE promotes an increase of the cytoplasmic level of the reporter gag mRNA that is in accord with elevated levels of Gag protein production. We also noted a reproducible difference in the increase of gag mRNA and Gag protein levels, suggesting that posttranscriptional regulation was affected at all steps from transport, stabilization to translation. This is in accord with previous observations [30-33] that posttranscriptional regulation of such mRNAs includes both export and translation.
Synergistic effect of RTEm26CTE on HIV-I env expression To rule out that the observed synergistic effect is a unique feature of the gag reporter mRNA, we inserted RTEm26CTE into an HIV-1 env reporter plasmid pNL1.5E (Figure 2A), expressing the authentic env cDNA from the HIV-1 LTR promoter. Like gag, env is poorly expressed (Figure 2B, lane 8 ) in the absence of a positive-acting export system, as expected. Both plasmids, containing either CTE (lanes 1,2 ) or RTE (lanes 3, 4) alone, showed $\sim 10 \times$ fold increase in Env production compared to the pNL1.5E (lane 8). The presence of RTEm26-CTE led to an additional increase in Env production (lane 5). A semi-quantitative analysis using serial dilutions (lanes 5-7) of the cell extract shows a $~ 100 \times$ fold activation, confirming synergistic effect of RTEm26-CTE. This expression level was comparable to that obtained in the presence of Rev (lanes 9-11). These data demonstrate that the synergistic effect of the combination of RTEm26-CTE export elements is applicable for different poorly expressed, unstable HIV-1 mRNAs.

\section{Synergistic effect of RTEm26CTE on expression of a Rev- and RRE-deficient HIV-I and SIV molecular clones}

To test the synergistic potency of the RTEm26-CTE in a more complex system, we inserted the combination element into the Rev- and RRE-minus molecular clones of HIV-1 NL4-3 (Figure 3) and SIVmac239 (Figure 4). Both of these viruses are unable to produce structural proteins or infectious virus in the absence of the viral Rev/RRE regulatory system $[6,11,12,19,23,34,35]$ (see also Figure 3B).

Upon insertion of CTE or RTE alone into the Rev- and RRE-minus NL4-3, we had previously shown that these RNA transport elements are able to partially replace the viral Rev-RRE system resulting in the production of infectious virus $[6,9,11,12]$ (Figure 3B and 3C). Interestingly, Western immunoblot analysis showed that the presence of RTEm26-CTE mediated a dramatic synergistic increase in expression of both env and gag compared to the clones containing each element alone (Figure 3B). Quantitation of gag expression using an antigen capture assay showed an increase of $\sim 1$ log over the presence of CTE or RTE alone. The expression level in the presence of RTEm26CTE was only slightly lower ( $\sim 3 x-$ fold) than those obtained by the wild type HIV-1 NL4-3 (Figure 3B). Upon infection of Jurkat cells, the RTEm26-CTE containing Revindependent HIV-1 clone as well as the RTE- or CTE-containing clones showed similar replicative capacities to levels $\sim 1 \log$ lower than that of the wild type HIV-1 (Figure $3 \mathrm{C})$. Thus, the presence of RTEm26-CTE is able to promote a balanced expression of the viral proteins able to generate infectious virus.

Similarly, we found that the presence of RTEm26-CTE also greatly increased expression of the Rev- and RREminus molecular clone of SIVmac239 (Figure 4B) to levels 

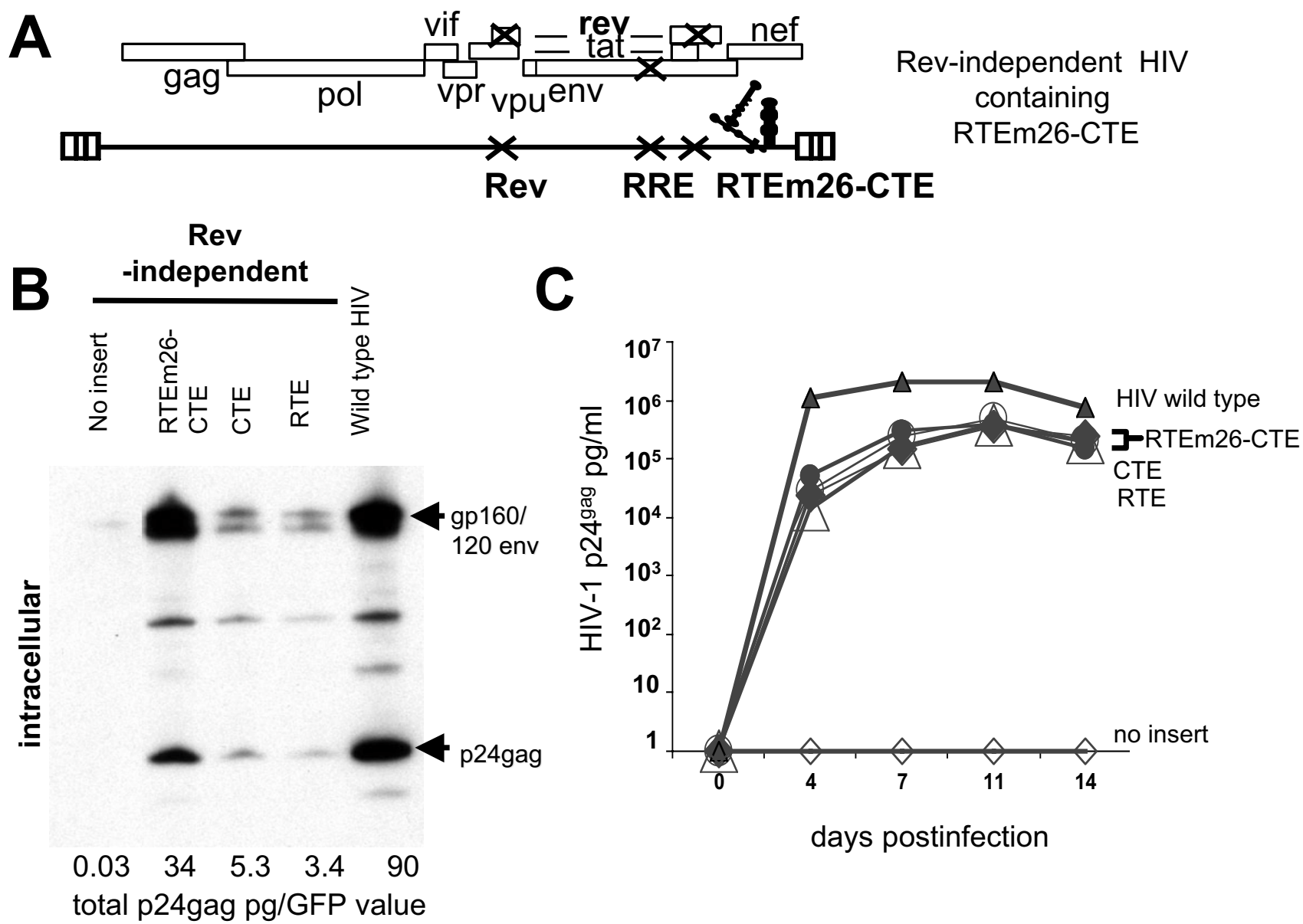

Figure 3

RTEm 26-CTE replaces Rev/RRE of HIV. A) Structure of rev and RRE-minus HIV-I containing RTEm26-CTE. Multiple point mutations inactivate both rev and RRE. CTE, RTE or RTEm26-CTE were inserted between env and the 3'LTR, rendering these clones nef-minus. B) Human 293 cells were transiently transfected with the indicated plasmids. Two days later, cell extracts were analyzed on Western immunoblots using HIV patient sera. Total intra- and extracellular Gag production was measured using commercial HIV p24 antigen capture assays and GFP production was quantitated. Normalized values (total gag in pg/total GFP units) are shown. C) HIV propagation in Jurkat cells. Transfected 293 cells were cocultivated with Jurkat cells: wild type NL4-3 (filled triangle), the Rev-independent HIV containing RTEm26-CTE (two clones filled diamond, open circle), CTE (open triangle), RTE (filled circle), and no insert (open diamond). Virus production was monitored over time using a commercial HIV p24gag antigen capture assay. Similarly, upon cell-free infection (not shown), the RTEm26-CTE replicates to a similar extent like the RTE- or CTE-containing Rev-independent HIV viruses.

about 10x-fold higher than those obtained by SIV clone containing only the CTE. Like its HIV counterpart, the RTEm26-CTE-containing SIV produces infectious virus (Figure 4C). We noted that it replicates with growth kinetics similar to the wild type SIV, in both CEMx174 cells (Figure 4C) and monkey PBMCs (data not shown), despite its slightly reduced level of expression (Figure 4B). In contrast to its HIV counterpart, the presence of the more potent RNA export element combination improved the replicative capacity when compared to the virus that contains only the CTE (compare peak at day 14 postinfection). Since we could not test propagation of SIV and HIV in the same cell types, it is possible that cellular factors may contribute to this phenomenon and this was not further investigated.

In conclusion, we have shown that the potent posttranscriptional effect of the RTEm26-CTE combination of RNA export elements from simple expression vectors (Figures 1 and 2) as well as from the complex array of mRNAs 


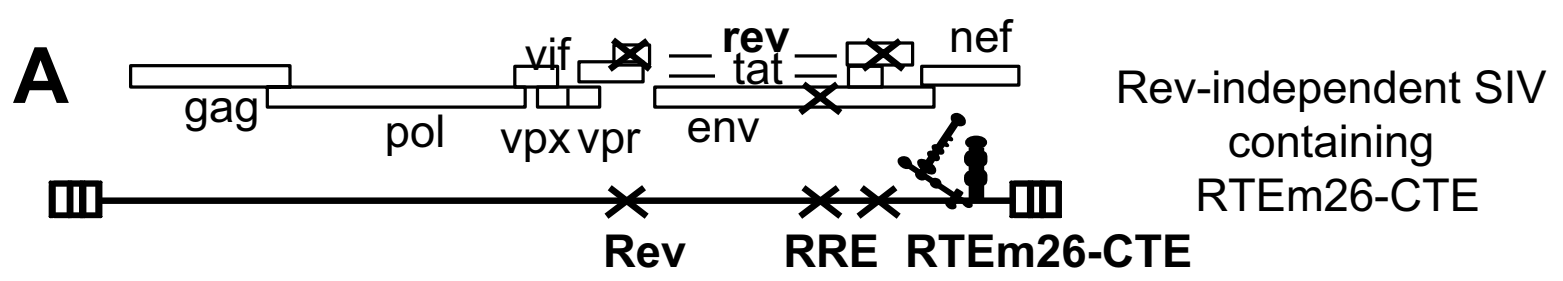

Rev
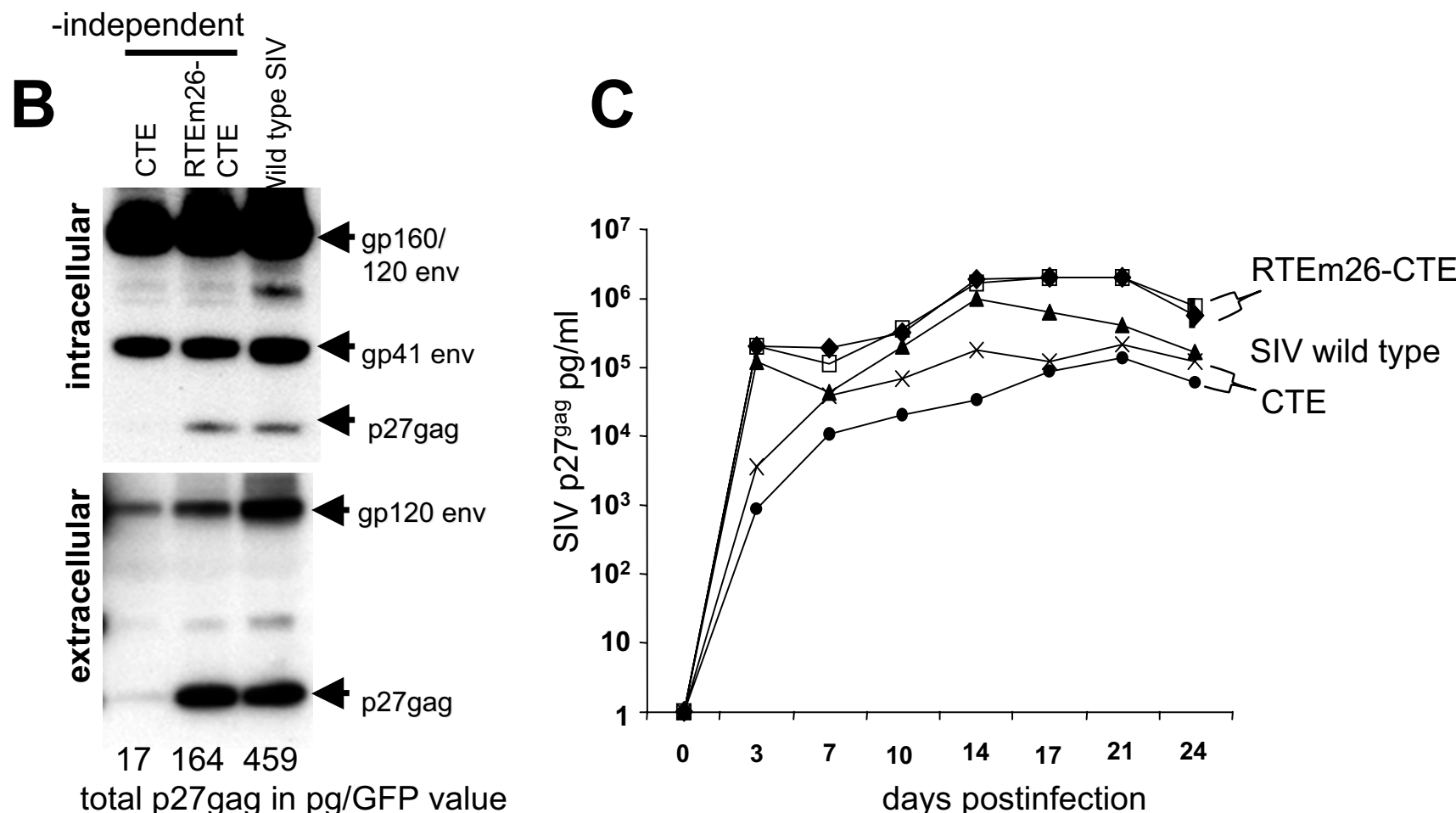

Figure 4

RTEm26-CTE replaces rev/RRE of SIV. A) Structure of the rev- and RRE-minus SIVmac239 containing RTEm26-CTE. Multiple point mutations inactivate both rev and RRE of SIVmac239. CTE or RTEm26-CTE was inserted between env and the 3'LTR. B) Human 293 cells were transiently transfected with the indicated plasmids. Two days later, cells and supernatant were analyzed for gag and env expression. Intracellular (I/I0 of cell extract) and extracellular (I/I50 of supernatant) were analyzed on Western immunoblots using a serum pool from SIV-infected monkeys. Total intra- and extracellular Gag production was measured using commercial SIV p27gag antigen capture assay and GFP production was quantitated. Normalized values (total gag in pg/total GFP units) are shown. C) SIV propagation in CEMxI74 cells. Virus stock were generated upon cocultivation of transfected 293 cells with CEMxI74 cells and then used to infect fresh CEMxI74 cells: wild type SIVmac239 (filled triangle), two stocks containing the Rev-independent SIV containing CTE (filled circle and X, respectively), and two stocks containing the RTEm26-CTE (filled diamond and open square, respectively). Virus production was monitored over time using a commercial SIV p27gag antigen capture assay.

produced from the molecular clones of HIV and SIV (Figures 3 and 4 ).

\section{Synergy depends on the spatial arrangement of RTEm26 and CTE}

To further understand the mechanism of the synergistic effect we generated a series of RTE-CTE containing plasmids with variations in the type of elements and their spatial arrangement. Since all our expression vectors utilize the $3^{\prime}$ LTR as polyadenylation signal, we first asked whether the choice of this signal could contribute to the synergistic effect. Replacing the HIV-1 polyadenylation signal with that of the bovine growth hormone had no effect (data not shown). Next, we tested the effect of wild type RTE instead of the up-regulatory mutant RTEm 26. Figure 5A shows that the substitution of RTEm 26 within the context of the combination element with the wild type RTE led to a $\sim 2$-fold lower expression. This reduction can 
A
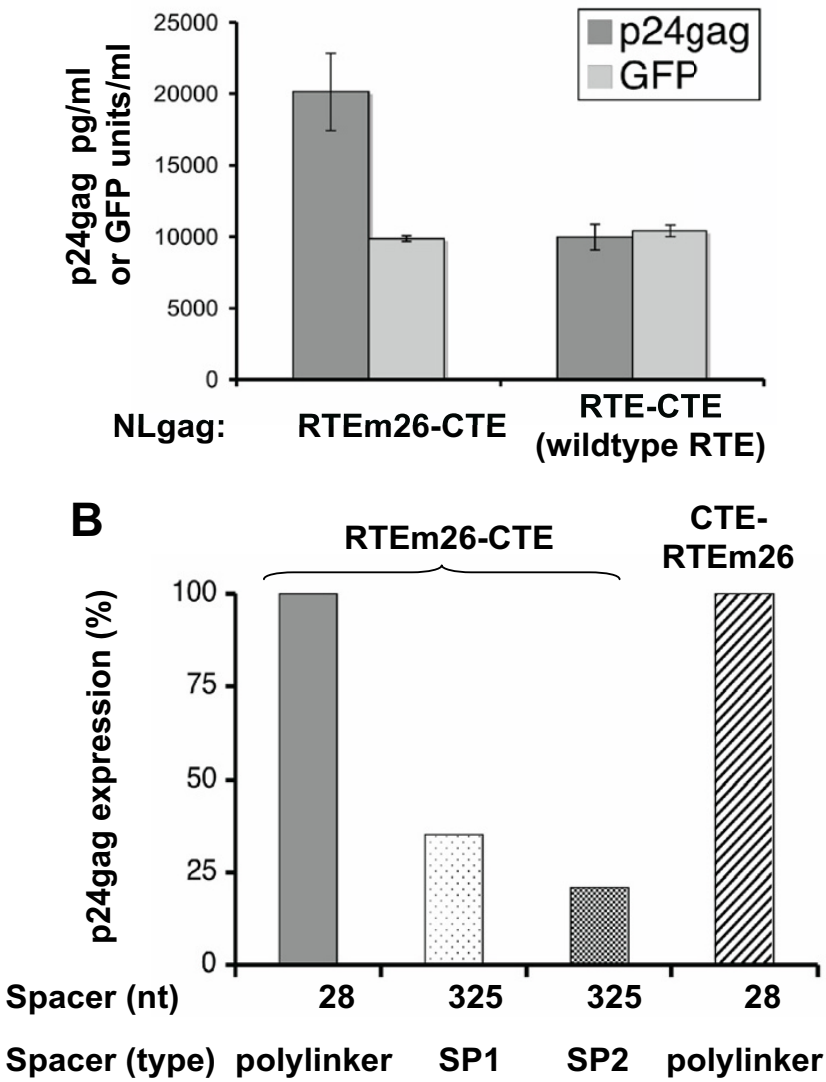

Figure 5

Optimal design of RTEm26-CTE combination element. A) Expression of pNLgag containing up-regulatory mutant RTEM 26 or the wild type RTE in combination with the CTE. HeLa cells were transfected with the independent clones of indicated plasmids and analyzed for Gag expression as described in Figure I. Standard deviations are shown. B) Organization of RTEm26-CTE element. pNLgag containing either RTEm26-CTE or the CTE-RTEm26, having the elements in reverse order separated by a $28-n t$ polylinker spacer, were analyzed. A spacer of $325 \mathrm{nt}$ from either a synthetic HIV-I tat gene (SPI) or from the cat gene (SP2) were inserted between RTEm 26 and CTE in pNLgagRTEm26-CTE. A typical experiment is shown using the average of two to four plasmids per construct. The data are presented in \% of Gag production by normalizing the values produced by pNLgagRTEm26-CTE to $100 \%$.

be explained by the $50 \%$ reduced activity of the wild type RTE compared to RTEm26 [7]. To further support the notion that active elements are required for synergy, we tested the combination of RTEm 26 and the inactive CTE (mutant CTEm36 [8]), which lacks the NXF1 binding site but maintains the overall secondary structure. This combination of elements showed activity similar to a single RTEm26 (data not shown). Therefore, to achieve maximal synergistic effect requires the presence of both elements in their most active form.

We then tested whether the spatial arrangement of RTEm 26 and CTE contributed to the synergistic effect. First, the reversal of the order of the elements from RTEm26-CTE to CTE-RTEm26 (Figure 5B) showed that the combination of the elements functions similarly in either configuration. Second, the $28 \mathrm{nt}$ spacer between the elements was increased by insertion of a 325-nt spacer sequence (SP1), which led to a significant loss of synergy (Figure 5B). To exclude that the nature of the spacer RNA contributed to this effect, a different RNA fragment (SP2) was used (Figure 5B), resulting in a similar decrease in gag expression. Separation of the elements by shorter spacers of 202 and $100 \mathrm{nt}$ led to gradual decrease in RTEm26-CTE activity (data not shown). Thus, the optimal synergistic effect requires the up-regulatory mutant RTE (RTEm26) and a functional CTE at close proximity.

The question arose whether multimers of CTE or RTE alone could achieve a similar effect. The presence of a CTE multimer has been reported to improve expression i.e. $4 \mathrm{xCTE}$ in a gag/pol reporter further elevated expression but this depended on the nature of the polyA signal [36], whereas multiple copies of the CTE had little or no effect in other mRNAs ([36], our own observations), suggesting that an effect of CTE multimers depended on the mRNA context. Using the gag reporter plasmid used herein, pNLgag, we found that two adjacent CTE elements also synergized reaching $\sim 75 \%$ of the effect observed for RTEm 26 CTE. In contrast, we found that RTEm 26 does not synergize with itself. Thus, while the effect of CTE multimers is transcript dependent, the RTEm26-CTE mediated synergistic increase in gene expression was persistently observed using different mRNAs (Figures 1, 2, 3, 4). Most importantly, the use of RTEm26-CTE has another great advantage, because this combination avoids the presence of adjacent repeated sequences, which may cause plasmid instability during growth in bacteria.

\section{Discussion}

The rather unexpected finding of this work was that the combination of two retroviral/retroelement-derived cisacting RNA export elements, RTE and CTE, synergistically increased expression of different retroviral mRNAs that are otherwise poorly expressed (Figures 1, 2, 3, 4). Since the function of RTEm26-CTE is conserved in mammalian cells, their integration into expression vectors provides a potent tool to improve expression of poorly expressed, unstable retroviral mRNAs to levels otherwise only achieved via more cumbersome RNA optimization.

Whereas the main restriction retroviral mRNAs encounter is their nucleocytoplasmic transport, other mRNAs may 
have different restrictions. The question arises whether the RTEm26-CTE combination has any effect on the expression of genes or cDNAs, which have posttranscriptional restrictions other than those of the lentiviral mRNAs. No improvement of expression was found using either the RTE, the CTE or the RTEm26-CTE combination in a MuLV-derived retroviral vector [37], while insertion of the woodchuck element WPRE augmented expression of this MuLV mRNA. These data indicate that oncoretrovirus and lentivirus expression have distinct restrictions. We further tested whether the presence of these RNA export elements could counteract posttranscriptional control that is not exerted at the RNA transport level but only involves cytoplasmic control. We noted that these export elements, as expected, did not alleviate the downregulatory effect of the AU-rich element (ARE)-containing IL-3 mRNA using the GFP-IL-3 hybrid mRNAs as a model system [38]. Thus, this finding supports the specific mechanism of function of the RTE, CTE and RTEm26-CTE, namely nucleocytoplasmic export. For this reason, we tested RTEm26-CTE effect upon insertion into some of our already RNA-optimized HIV and SIV gag and env cDNAs vectors, whose mRNAs are efficiently exported leading to very high expression in cultured cells. As expected, we only found a less than 2-fold effect on this already optimized mRNAs. It remains to be tested whether export signals like the RTEm26-CTE could act as additional positive acting signals and mediate higher expression levels in primary cells, for example upon DNA vaccination of animals or using gene transfer vectors.

NXF1 provides a key molecular link between mRNA and components of the nuclear pore complex. A possible model to explain the synergistic effect of RTE and CTE is that the duplication of these export elements may provide an improved target for NXF1 resulting in more efficient nucleocytoplasmic mRNA transport. Using in vitro gelshift assays, we found that the binding of NXF1 (aa 61372) to radiolabeled CTE is competed similarly by both excess cold CTE as well as RTEm26-CTE RNAs (data not shown). These data indicate that NXF1 binds to CTE as well as to the RTEm26-CTE RNA targets with similar affinity. We have previously shown that NXF1 is not a high affinity binder of RTE when compared to the CTE [6], suggesting the role of a distinct cellular protein mediating RTE RNA export. It is plausible that this putative factor tethers the RTE-RNAs directly or indirectly to the NXF1 pathway. Therefore, it is likely that the putative RTE-binding protein and NXF1 may act cooperatively. Studies are on-going to delineate the detailed mechanism of function mediating this cooperativity.

mRNA expression is controlled at several steps including nuclear export, cytoplasmic trafficking and polysomal association. The use of strong mRNA export elements is a powerful tool to alleviate restrictions linked to nuclear export. For a subset of lentiviral mRNAs encoding gag, pol or env, posttranscriptional control has been shown at multiple steps of export and polysomal association. The presence of potent RNA export elements is sufficient to alleviate all of theses restrictions. Integration of RTEm26CTE into lentiviral vectors will increase gene expression essential for applications such as in gene therapy that are otherwise only obtained through RNA optimization. For DNA-based vaccine vectors, it remains to be seen whether the presence of strong binding sites for the cellular mRNA transport machinery is of further advantage when introduced into primary tissues in animals as compared to cultured cells. In addition, these retroviral/retroelement derived RNA export elements provide unique tools to further dissect mechanisms involved in posttranscriptional regulation of viral and cellular genes.

\section{Conclusion}

The use of the combination of RNA export elements, comprising the up-regulatory mutant RTEm 26 and the CTE, potently increase lentiviral gene expression.

\section{Methods \\ Plasmids}

The RTE, RTEm26, and CTE were inserted into pNLgag $[23,24,39]$ between the gag gene and the 3 'LTR and have been described [7]. RTE or RTEm 26 was inserted into the SacII site located $5^{\prime}$ to the CTE, generating pNLgagRTECTE and pNLgagRTEm26-CTE, respectively. In pNLgag RTEm26-SP1-CTE, a spacer sequence (SP1) of $325 \mathrm{nt}$ from a synthetic HIV-1 tat gene (BamHI-XbaI from plasmid $32 \mathrm{H}$ ) was inserted between RTEm 26 and CTE. In pNLgag RTEm26-SP2-CTE, a spacer (SP2) from a different source (cat gene) of $325 \mathrm{nt}$ was inserted. Similarly spacers or 202 and $100 \mathrm{nt}$ were inserted. The bovine growth hormone polyadenylation signal was inserted between Sall and XhoI sites 3' to RTEm26-CTE replacing the 3'LTR. pNLcgag [24] is similar to pNLgag, except it lacks the major splice donor. pNL1.5E expresses the authentic HIV-1 env cDNA from the LTR promoter [40]. RTE, CTE and RTEm26-CTE were inserted as $S m a I-X h o I$ fragment between the env gene and the 3' LTR into BlpI and XhoI digested pNL1.5E. The Rev-independent clones of NL4-3 [RRE(-)Rev(-), RRE()Rev(-)CTE, and RRE(-)Rev(-)RTE] have been published previously $[6,12,41]$. RTEm26-CTE was inserted into the XhoI site of the RRE(-)Rev(-) NL4-3. The SIVmac239 RRE(-)Rev(-)nefdelCTE is similar to the published SIVmac239 RRE(-)Rev(-)Nef(-)CTE [35] but contains an additional deletion of the remaining nef region 3 ' to the CTE [42]. RTEm26-CTE was inserted in the place of CTE. The GFP-IL-3 plasmid contains the IL3 3'UTR inserted 3' to the enhanced green fluorescent protein (GFP) gene in pFRED25 [43]. RTEm26, CTE, or RTEM26CTE were inserted between GFP and the 3'UTR. These elements were 
further inserted between the cDNAs and the polyadenylation signals of vectors expressing the RNA-optimized HIV1 env $(75 \mathrm{H})$.

\section{Transfections}

Human HLtat, a HeLa-derivative producing HIV tat [44] or human 293 cells were transfected with $1 \mu \mathrm{g}$ of the NLgag plasmids. HLtat provides Tat to activate gene expression from the viral LTR promoter. For transfection of 293 cells a tat expression plasmid, pBstat, was also cotransfected. We routinely analyzed $2-3$ independent clones in duplicate determinations. Two to three days later, the cell extracts were analyzed for Gag expression using a commercial HIV-1 p24gag or the SIV p27gag antigen capture assay. Gag and Env production was also analyzed on Western immunoblot using plasma from HIV-1 infected persons, rabbit anti-HIV-1 env serum or SIVmac infected rhesus macaques [23]. Cotransfection of $0.8 \mu \mathrm{g}$ of the GFP expression vector pFRED25 [43] served as internal control. Cotransfection of the secreted version of alkaline phosphatase SEAP [45] as internal control was used in some experiments and SEAP levels were determined from the culture supernatant using a commercial kit (Tropix, Inc.). Transfections of 293 cells were performed using FUGENE-6, whereas the Calcium-phosphate coprecipitation technique was used for HeLa cells. GFP-IL3 plasmids were transfected into NIH3T3 cells and analyzed by fluorescent activated cell sorting (FACS) as described [38]. Total and cytoplasmic polyadenylated mRNA was isolated and analyzed as described $[12,46]$. Hybridization of the blots with a GFP probe was used to evaluate transfection and RNA extraction efficiency. Blots were quantitated using the STORM860 phosphoimager.

\section{Abbreviations}

CTE, constitutive Transport Element; RTE, RNA Transport Element; RRE, Rev-Responsive Element; HIV-1, human immunodeficiency virus type 1 ; SIV, simian immunodeficiency virus; IAP, intracisternal A-particle retroelement; SRV/D, simian type D retroviruses; NXF1, nuclear export factor 1 .

\section{Competing interests}

The author(s) declare that they have no competing interests.

\section{Authors' contributions}

SS generated RTEM26-CTE constructs and performed expression studies; RJ, MR, AvG provided additional constructs and performed expression studies; DM performed in vitro binding studies; JB, CA performed experiments in using infectious HIV and SIV and provided technical assistance; ASZ and CM provided reagents and intellectual input; GNP provided intellectual input and contributed to the manuscript; BKF directed the project and wrote the manuscript.

\section{Acknowledgements}

We thank S. Lindtner for comments, our Werner H. Kirsten Student Intern program recipients C. Jodrie, A. Gainer, L. Kotani, T. Hudzik, and S. Sadtler for their contributions, L. Arthur and J. Lifson for antiserum, M. Lu for technical assistance, and T. Jones for editorial assistance. AvG and MR are contractors through SAIC. This research was supported by the Intramural Research Program of the National Institutes of Health, National Cancer Institute.

\section{References}

I. Grüter P, Tabernero C, von Kobbe C, Schmitt C, Saavedra C, Bachi A, Wilm M, Felber BK, Izaurralde E: TAP, the human homolog of Mex67p, mediates CTE-dependent RNA export from the nucleus. Mol Cell 1998, I:649-659.

2. Segref A, Sharma K, Doye V, Hellwig A, Huber J, Luhrmann R, Hurt $\mathrm{E}:$ Mex67p, a novel factor for nuclear mRNA export, binds to both poly(A)+ RNA and nuclear pores. Embo J I997, I 6( I I):3256-327I.

3. Tan W, Zolotukhin AS, Bear J, Patenaude DJ, Felber BK: The mRNA export in C. elegans is mediated by Ce-NXF-I, an ortholog of human TAP and S cerevisiae Mex67p. RNA 2000, 6: $1762-1772$.

4. Braun IC, Rohrbach E, Schmitt C, Izaurralde E: TAP binds to the constitutive transport element through a novel RNA-binding motif that is sufficient to promote CTE-dependent RNA export from the nucleus. EMBO J 1999, I 8:1953-1965.

5. Erkmann JA, Kutay U: Nuclear export of mRNA: from the site of transcription to the cytoplasm. Exp Cell Res 2004, 296( I): 12-20.

6. Nappi F, Schneider R, Zolotukhin AS, Smulevitch S, Michalowski D, Bear J, Felber BK, Pavlakis GN: Identification of a novel posttranscriptional regulatory element using a rev and RRE mutated HIV-I DNA proviral clone as a molecular trap. J Virol 200I, 75(I 0):4558-4569.

7. Smulevitch S, Michalowski D, Zolotukhin AS, Schneider R, Bear J, Roth P, Pavlakis GN, Felber BK: Structural and Functional Analysis of the RNA Transport Element (RTE), a Member of an Extensive Family Present in the Mouse Genome. J Virol 2005, 79:2356-2365.

8. Tabernero C, Zolotukhin AS, Valentin A, Pavlakis GN, Felber BK: The posttranscriptional control element of the simian retrovirus type I forms an extensive RNA secondary structure necessary for its function. J Virol 1996, 70:5998-60 II.

9. Tabernero C, Zolotukhin AS, Bear J, Schneider R, Karsenty G, Felber BK: Identification of an RNA Sequence Within an Intracisternal-A Particle Element Able to Replace Rev-Mediated Posttranscriptional Regulation of Human Immunodeficiency Virus Type I. J Virol 1997, 71:95-101.

10. Ernst RK, Bray M, Rekosh D, Hammarskjold ML: Secondary structure and mutational analysis of the Mason-Pfizer monkey virus RNA constitutive transport element. RNA 1997, 3:210-222.

II. Bray M, Prasad S, Dubay JW, Hunter E, Jeang KT, Rekosh D, Hammarskjold ML: A small element from the Mason-Pfizer monkey virus genome makes human immunodeficiency virus type I expression and replication Rev-independent. Proc Natl Acad Sci USA 1994, 91:1256-1260.

12. Zolotukhin AS, Valentin A, Pavlakis GN, Felber BK: Continuous propagation of $\operatorname{RRE(-)}$ and $\operatorname{Rev}(-) \operatorname{RRE}(-)$ human immunodeficiency virus type I molecular clones containing a cis-acting element of simian retrovirus type I in human peripheral blood lymphocytes. J Virol 1994, 68:7944-7952.

13. Ernst RK, Bray M, Rekosh D, Hammarskjold ML: A structured retroviral RNA element that mediates nucleocytoplasmic export of intron-containing RNA. Mol Cell Biol 1997, I 7:| 35-I 44.

14. Izaurralde E, Spector DL: Nucleus and gene expression; The interplay of transcriptional and post-transcriptional mechanisms that regulate gene expression. Curr Opin Cell Biol 2004, I 6(3):219-222. 
I5. Dreyfuss G, Kim VN, Kataoka N: Messenger-RNA-binding proteins and the messages they carry. Nat Rev Mol Cell Biol 2002, 3(3): 195-205.

16. Vinciguerra $P$, Stutz F: mRNA export: an assembly line from genes to nuclear pores. Curr Opin Cell Biol 2004, I 6(3):285-292.

17. Malim MH, Hauber J, Le SY, Maizel JV, Cullen BR: The HIV-I rev trans-activator acts through a structured target sequence to activate nuclear export of unspliced viral mRNA. Nature 1989, 338:254-257.

18. Hammarskjöld ML, Heimer J, Hammarskjöld B, Sangwan I, Albert L, Rekosh D: Regulation of human immunodeficiency virus env expression by the rev gene product. J Virol 1989, 63:1959-1966.

19. Feinberg MB, Jarrett RF, Aldovini A, Gallo RC, Wong-Staal F: HTLVIII expression and production involve complex regulation at the levels of splicing and translation of viral RNA. Cell 1986 46:807-8I7.

20. Schwartz S, Felber BK, Pavlakis GN: Distinct RNA sequences in the gag region of human immunodeficiency virus type I decrease RNA stability and inhibit expression in the absence of Rev protein. J Virol 1992, 66:150-159.

21. Schneider R, Campbell M, Nasioulas G, Felber BK, Pavlakis GN: Inactivation of the human immunodeficiency virus type I inhibitory elements allows Rev-independent expression of Gag and Gag/Protease and particle formation. J Virol 1997, 7 I:4892-4903

22. Cochrane AW, Jones KS, Beidas S, Dillon PJ, Skalka AM, Rosen CA Identification and characterization of intragenic sequences which repress human immunodeficiency virus structural gene expression. J Virol 1991, 65(10):5305-53 I3.

23. Hadzopoulou-Cladaras M, Felber BK, Cladaras C, Athanassopoulos $A$, Tse $A$, Pavlakis GN: The rev (trs/art) protein of human immunodeficiency virus type I affects viral mRNA and protein expression via a cis-acting sequence in the env region. Virol 1989, 63:1265-1274.

24. Felber BK, Hadzopoulou-Cladaras M, Cladaras C, Copeland T, Pavlakis GN: rev protein of human immunodeficiency virus type I affects the stability and transport of the viral mRNA. Proc Natl Acad Sci USA 1989, 86:|495-1499.

25. Nasioulas G, Zolotukhin AS, Tabernero C, Solomin L, Cunningham $\mathrm{CP}$, Pavlakis GN, Felber BK: Elements distinct from human immunodeficiency virus type I splice sites are responsible for the Rev dependence of env mRNA. I Virol 1994 68(5):2986-2993.

26. Maldarelli F, Martin MA, Strebel K: Identification of posttranscriptionally active inhibitory sequences in human immunodeficiency virus type I RNA: Novel level of gene regulation. J Virol 1991, 65:5732-5743.

27. Schwartz S, Campbell M, Nasioulas G, Harrison J, Felber BK, Pavlakis GN: Mutational inactivation of an inhibitory sequence in human immunodeficiency virus type-I results in Rev-independent gag expression. J Virol 1992, 66:7176-7|82.

28. Suh D, Seguin B, Atkinson S, Ozdamar B, Staffa A, Emili A, Mouland $A$, Cochrane $A$ : Mapping of determinants required for the function of the HIV-I env nuclear retention sequence. Virology 2003, 3 I 0(I):85-99.

29. Lu X, Lewis N, Rekosh D, Hammarskjold ML: A 5' splice site is essential for rev and rex regulation of HIV envelope protein mRNA expression. In Advances in Molecular Biology and Targeted Treatment for AIDS Edited by: Kumar A. New York, Plenum; I99I.

30. Dangel AW, Hull S, Roberts TM, Boris-Lawrie K: Nuclear interactions are necessary for translational enhancement by spleen necrosis virus RU5. J Virol 2002, 76(7):3292-3300.

31. Jin L, Guzik BW, Bor YC, Rekosh D, Hammarskjold ML: Tap and NXT promote translation of unspliced mRNA. Genes Dev 2003, I 7(24):3075-3086.

32. Hull S, Boris-Lawrie K: RU5 of Mason-Pfizer monkey virus 5' long terminal repeat enhances cytoplasmic expression of human immunodeficiency virus type I gag-pol and nonviral reporter RNA. J Virol 2002, 76(20): $10211-10218$.

33. D'Agostino DM, Felber BK, Harrison JE, Pavlakis GN: The Rev protein of human immunodeficiency virus type I promotes polysomal association and translation of gag/pol and vpu/env mRNA. Mol Cell Biol 1992, I2:1375-1386.

34. Sodroski J, Goh WC, Rosen C, Dayton A, Terwilliger E, Haseltine W: $A$ second post-transcriptional trans-activator gene required for HTLV-III replication. Nature 1986, 32 I:4I 2-4I7.
35. von Gegerfelt AS, Felber BK: Replacement of posttranscriptional regulation in SIVmac239 generated a Rev-independent infectious virus able to propagate in rhesus peripheral blood mononuclear cells. Virology 1997, 232:291-299.

36. Wodrich H, Bohne J, Gumz E, Welker R, Krausslich HG: A new RNA element located in the coding region of a murine endogenous retrovirus can functionally replace the Rev/Revresponsive element system in human immunodeficiency virus type I Gag expression. J Virol 200I, 75(22): |0670- I0682.

37. Hlavaty J, Schittmayer M, Stracke A, Jandl G, Knapp E, Felber BK, Salmons B, Gunzburg WH, Renner M: Effect of posttranscriptional regulatory elements on transgene expression and virus production in the context of retrovirus vectors. Virology 2005 , $34|(I):|-\mid I$.

38. Benjamin D, Colombi M, Moroni C: A GFP-based assay for rapid screening of compounds affecting ARE-dependent mRNA turnover. Nucleic Acids Res 2004, 32(I I):e89.

39. Solomin L, Felber BK, Pavlakis GN: Different sites of interaction for Rev, Tev, and Rex proteins within the Rev responsive element of human immunodeficiency virus type I. J Virol 1990, 64:6010-6017.

40. Schwartz S, Felber BK, Fenyö EM, Pavlakis GN: Env and Vpu proteins of human immunodeficiency virus type I are produced from multiple bicistronic mRNAs. J Virol 1990, 64:5448-5456.

4I. Valentin A, Aldrovandi G, Zolotukhin AS, Cole SW, Zack JA, Pavlakis GN, Felber BK: Reduced viral load and lack of CD4 depletion in SCID-hu mice infected with Rev-independent clones of human immunodeficiency virus type I. J Virol 1997, 71:9817-9822.

42. von Gegerfelt AS, Alicea C, Valentin A, Van Rompay KKA, Markham P, Marthas ML, Pavlakis GN, Ruprecht RM, Felber BK: Long lasting control and lack of pathogenicity of the attenuated Rev-independent SIV in rhesus macaques. submitted

43. Stauber RH, Horie K, Carney P, Hudson EA, Tarasova NI, Gaitanaris GA, Pavlakis GN: Development and applications of enhanced green fluorescent protein mutants. Biotechniques 1998 , 24(3):462-6, 468-7।

44. Schwartz S, Felber BK, Benko DM, Fenyö EM, Pavlakis GN: Cloning and functional analysis of multiply spliced mRNA species of human immunodeficiency virus type I. J Virol 1990, 64:2519-2529.

45. Berger J, Hauber J, Hauber R, Geiger R, Cullen BR: Secreted placental alkaline phosphatase: a powerful new quantitative indicator of gene expression in eukaryotic cells. Gene 1988 , 66: $1-10$.

46. Zolotukhin AS, Tan W, Bear J, Smulevitch S, Felber BK: U2AF participates in the binding of TAP (NXFI) to mRNA. J Biol Chem 2002, 277(6):3935-3942. 